\title{
MEASUREMENT OF THE ISOTOPIC COMPOSITION OF HYDROGEN AND HELIUM NUCLEI IN COSMIC RAYS WITH THE PAMELA-EXPERIMENT
}
W. Menn ${ }^{* 19}$, O. Adriani ${ }^{5,6}, \mathrm{G}$
G. C. Barbarino 7,8 ,
G. A. Bazilevskaya ${ }^{9}$, R. Bellotti ${ }^{10,11}$,
M. Boezio ${ }^{2}$, E. A. Bogomolov ${ }^{12}$, M. Bongi ${ }^{4,6}$, V. Bonvicini ${ }^{2}$, S. Bottai ${ }^{6}$, A. Bruno ${ }^{10,11}$,
F. Cafagna ${ }^{11}$, D. Campana ${ }^{8}$, P. Carlson ${ }^{13}$, M. Casolino ${ }^{3,15}$, G. Castellini ${ }^{16}$,
C. De Donato ${ }^{3}$, C. De Santis ${ }^{14}$, N. De Simone ${ }^{3}$, V. di Felice ${ }^{3,4}$ V. Formato ${ }^{1,2}$,
A. M. Galper ${ }^{17}$, A. V. Karelin ${ }^{17}$, S. V. Koldashov ${ }^{17}$, S. Koldobskiy ${ }^{17}$, S. Y. Krutkov ${ }^{12}$,

A. N. Kvashnin ${ }^{9}$, A. Leonov ${ }^{17}$,V. Malakhov ${ }^{17}$, L. Marcelli ${ }^{14}$, M. Martucci ${ }^{14,18}$,

A. G. Mayorov ${ }^{17}$, M Merge ${ }^{3,14}$, V. V. Mikhailov $^{17}$,E. Mocchiutti ${ }^{2}$, A. Monaco ${ }^{10,11}$, N. Mori ${ }^{6}$, R. Munini ${ }^{1,2}$, G. Osteria ${ }^{8}$, F. Palma ${ }^{3,14}$, B. Panico ${ }^{8}$ P. Papini $^{6}$, M. Pearce ${ }^{13}$, P. Picozza ${ }^{3,14}$, M. Ricci ${ }^{18}$, S. B. Ricciarini ${ }^{16}$, R. Sarkar ${ }^{2}$, V. Scotti ${ }^{7,8}$, M. Simon $^{19}$,

R. Sparvoli ${ }^{3,14}$, P. Spillantini ${ }^{5,6}$, Y. I. Stozhkov ${ }^{9}$, A. Vacchi $^{2}$, E. Vannuccini ${ }^{6}$, G. Vasilyev ${ }^{12}$, S. A. Voronov ${ }^{17}$, Y. T. Yurkin ${ }^{17}$, G. Zampa² ${ }^{2}$ N. Zampa ${ }^{2}$, M. S. Potgieter ${ }^{21}$, E.E. Vos ${ }^{21}$.

${ }^{1}$ University of Trieste, Department of Physics, I-34147 Trieste, Italy

${ }^{2}$ INFN, Sezione di Trieste I-34149 Trieste, Italy

${ }^{3}$ INFN, Sezione di Rome "Tor Vergata”, I-00133 Rome, Italy

${ }^{4}$ Agenzia Spaziale Italiana (ASI) Science Data Center, I-00044 Frascati, Italy

${ }^{5}$ University of Florence, Department of Physics, I-50019 Sesto Fiorentino, Florence, Italy

${ }^{6}$ INFN, Sezione di Florence, I-50019 Sesto Fiorentino, Florence, Italy

7 University of Naples “Federico II”, Department of Physics, I-80126 Naples, Italy

${ }^{8}$ INFN, Sezione di Naples, I-80126 Naples, Italy

${ }^{9}$ Lebedev Physical Institute, RU-119991, Moscow, Russia

${ }^{1} 0$ University of Bari, Department of Physics, I-70126 Bari, Italy

${ }^{11}$ INFN, Sezione di Bari, I-70126 Bari, Italy

12 Ioffe Physical Technical Institute, RU-194021 St. Petersburg, Russia

${ }^{13}$ KTH, Department of Physics, and the Oskar Klein Centre for Cosmoparticle Physics, AlbaNova University Centre, SE-10691 Stockholm, Sweden

${ }^{14}$ University of Rome "Tor Vergata”, Department of Physics, I-00133 Rome, Italy

${ }^{15}$ RIKEN, Advanced Science Institute, Wako-shi, Saitama, Japan

${ }^{16}$ IFAC, I-50019 Sesto Fiorentino, Florence, Italy

${ }^{17}$ National Research Nuclear University MEPhI, RU-115409 Moscow

18 INFN, Laboratori Nazionali di Frascati, Via Enrico Fermi 40, I-00044 Frascati, Italy

${ }^{19}$ Universität Siegen, Department of Physics, D-57068 Siegen, Germany

${ }^{20}$ INFN, Sezione di Perugia, I-06123 Perugia, Italy

E-mail: mennepamela.physik.uni-siegen.de 
The cosmic-ray hydrogen and helium $\left({ }^{1} \mathrm{H},{ }^{2} \mathrm{H},{ }^{3} \mathrm{He},{ }^{4} \mathrm{He}\right)$ isotopic composition between $100 \mathrm{MeV} / \mathrm{n}$ and $1.4 \mathrm{GeV} / \mathrm{n}$ has been measured with the satellite-borne experiment PAMELA, which was launched into low-Earth orbit on-board the Resurs-DK1 satellite on June $15^{\text {th }} 2006$. The rare isotopes ${ }^{2} \mathrm{H}$ and ${ }^{3} \mathrm{He}$ in cosmic rays are believed to originate mainly from the interaction of high energy protons and helium with the galactic interstellar medium. The energy spectrum of these components carries fundamental information regarding the propagation of cosmic rays in the galaxy which are competitive with those obtained from other secondary to primary measurements such as $\mathrm{B} / \mathrm{C}$. The isotopic composition was measured between 100 and $1100 \mathrm{MeV} / \mathrm{n}$ for hydrogen and between 100 and $1400 \mathrm{MeV} / \mathrm{n}$ for helium isotopes using two different detector systems over the $23^{\text {rd }}$ solar minimum from July 2006 to December 2007.

The 34th International Cosmic Ray Conference,

30 July- 6 August, 2015

The Hague, The Netherlands

${ }^{*}$ Speaker. 


\section{Introduction}

The rare isotopes ${ }^{2} \mathrm{H}$ and ${ }^{3} \mathrm{He}$ in cosmic rays are generally believed to be of secondary origin, resulting mainly from the nuclear interactions of primary cosmic-ray protons and ${ }^{4} \mathrm{He}$ with the interstellar medium. The spectral shape and composition of the secondary isotopes is therefore completely determined by the source spectrum of the parent elements and by the propagation process. Measurements of the secondary isotopes spectra are then a powerful tool to constrain the parameters of the galactic propagation models [8]. In the eighties and nineties there have been several measurements of stratospheric balloon experiments using superconducting magnetic spec-

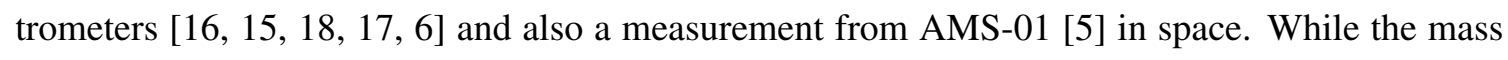
resolution of the balloon experiments was usually quite good, the residual atmosphere above the instruments caused a non-negligible background of secondary particles, their estimation is subject to large uncertainties (e.g. the limited knowledge of isotope production cross sections).

The PAMELA experiment has been observing galactic cosmic rays since July 2006 at an altitude ranging from $\sim 350 \mathrm{~km}$ to $\sim 600 \mathrm{~km}$ on-board of the Russian Resurs-DK1 satellite which executes a quasi-polar orbit $\left(70^{\circ}\right.$ inclination). The low-earth orbit allows PAMELA to perform measurements in an environment free from the background induced by interactions of cosmic rays within the atmosphere.

The results presented here are based on the data set collected by PAMELA between July 2006 and December 2007. This period provided us with a huge and unprecedented amount of data.

In this work we refer repeatedly to a previous PAMELA paper [四] which dealt with the same isotopes ${ }^{1} \mathrm{H},{ }^{2} \mathrm{H},{ }^{3} \mathrm{He}$ and ${ }^{4} \mathrm{He}$, but used solely the combination of velocity measurement provided by the ToF system in combination with the momentum measurement of the magnetic spectrometer. In this work here a more complete and elaborated fitting procedure (see chapter B.3.1) was employed, combined with a more stringent selection on cuts and on efficiencies. In addition we make use of the multiple energy loss measurements provided by the 44 planes of the imaging calorimeter. This not only allowed a cross check between the two techniques of isotopic separation within the PAMELA instrument (ToF and multiple $d E / d x$ versus rigidity) the multiple $d E / d x$ technique also allowed to extend the measurements for isotopes to higher energies.

\section{The PAMELA instrument}

The PAMELA satellite-borne cosmic ray experiment was built to measure charged particles in the cosmic radiation with a particular focus on antiparticles. It was mounted on the Resurs DK1 satellite and launched from the Baikonur cosmodrome on June 15th 2006. The PAMELA apparatus is composed of several sub-detectors: ToF system, anti-coincidence system (CARD, CAS, CAT), magnetic spectrometer with microstrip silicon tracking system, W/Si electromagnetic imaging calorimeter, shower-tail-catcher scintillator (S4) and neutron detector. A detailed description of the PAMELA instrument and an overview of the mission can be found in [4]]]. The core of the instrument is a magnetic spectrometer, made of a permanent magnet $(0.43 \mathrm{~T})$ and a silicon tracking system (resolution in the bending side $4 \mu \mathrm{m}$ ) for a maximum detectable rigidity of $1 \mathrm{TV}$. The momentum resolution is better than $4 \%$ between $2 \mathrm{GV}$ and $20 \mathrm{GV}$. The W/Si sampling imaging calorimeter comprises 44 single-sided silicon strip detector planes interleaved with 22 plates of 
tungsten absorber [ [ $]$ ]. Each tungsten layer has a thickness of 0.74 radiation lengths $(2.6 \mathrm{~mm})$ and it is sandwiched between two printed circuit boards, which house the silicon detectors as well as the frontend and digitizing electronics. Each silicon plane consists of $3 \times 3,380 \mu \mathrm{m}$ thick, $8 \times 8 \mathrm{~cm}^{2}$ detectors, segmented into 32 strips with a pitch of $2.4 \mathrm{~mm}$. The orientation of the strips for two consecutive silicon planes is shifted by 90 degrees, thus providing 2-dimensional spatial information. The total depth of the calorimeter is 16.3 radiation lengths and 0.6 nuclear interaction lengths.

\section{Data analysis}

\subsection{Event selection}

Each triggered event had to fulfill several criteria to be used for further analysis: Requirements were set on the event quality to select positively charged particles with a precise measurement of the absolute value of the particle rigidity and velocity. Galactic events were selected if the particles rigidity exceeded the critical rigidity, $\rho_{c}$, defined as 1.3 times the cutoff rigidity $\rho_{S V C}$ computed in the Störmer vertical approximation. The particle charge was identified using the ionization measurements provided by the silicon sensors of the magnetic spectrometer. Depending on the number of hit sensors there can be up to $12 d E / d x$ measurements. A rigidity dependent selection on the arithmetic mean of those measurements is used to select $Z=1$ or $Z=2$ candidates. The requirements are identical to the selection in [四] and we refer to this paper for more details.

\subsection{Isotope separation in the PAMELA instrument}

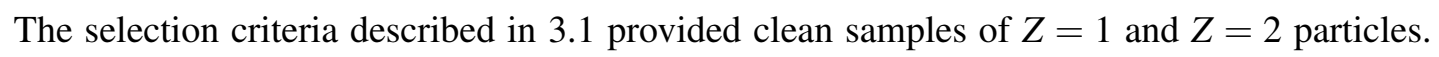
In each sample an isotopic separation at fixed rigidity is possible since the mass of each particle follows the relation $m=R Z e / \gamma \beta c$, where $R$ is the magnetic rigidity, $Z \times e$ is the particle charge, and $\gamma$ is the Lorentz factor. The particle velocity $\beta$ can either be provided directly from the timing measurement of the ToF system, or indirectly from the energy loss in the calorimeter, which follows $\beta$ via the Bethe-Bloch formula $d E / d x \propto \frac{Z^{2}}{\beta^{2}}$ (neglecting logarithmic terms).

\subsubsection{Isotope separation using ToF vs. rigidity}

For the ToF analysis we can use directly the $\beta$ provided by the timing measurement. In Fig. $\mathbb{W}$ we show $\beta$ vs. the particle rigidity for $Z=1$ and $Z=2$ data. The black lines in the figure represent the expectations for each isotope.

\subsubsection{Isotope separation using multiple $d E / d x$ in the calorimeter vs. rigidity}

The isotopic analysis of nuclei with the calorimeter is restricted to events which do not interact inside the calorimeter. To check if an interaction occurs, we derive in each silicon layer a) the total energy detected $\left(q_{t o t}\right)$ and b) the energy deposited in the strip closest to the track and the neighbouring strip on each side $\left(q_{\text {track }}\right)$. In the ideal case the fraction of $q_{\text {track }} / q_{\text {tot }}$ will be equal to one, a value less then one means that strips outside the track were hit. Starting from the top of the calorimeter we calculate $\Sigma q_{\text {track }} / \Sigma q_{\text {tot }}$ at each layer, as long as this value is greater than 0.9 , we use these layers for further analysis. In this way we can make use of slow particles, which stop early in the calorimeter, particles which interact somewhere, but also all clean events with the particle 

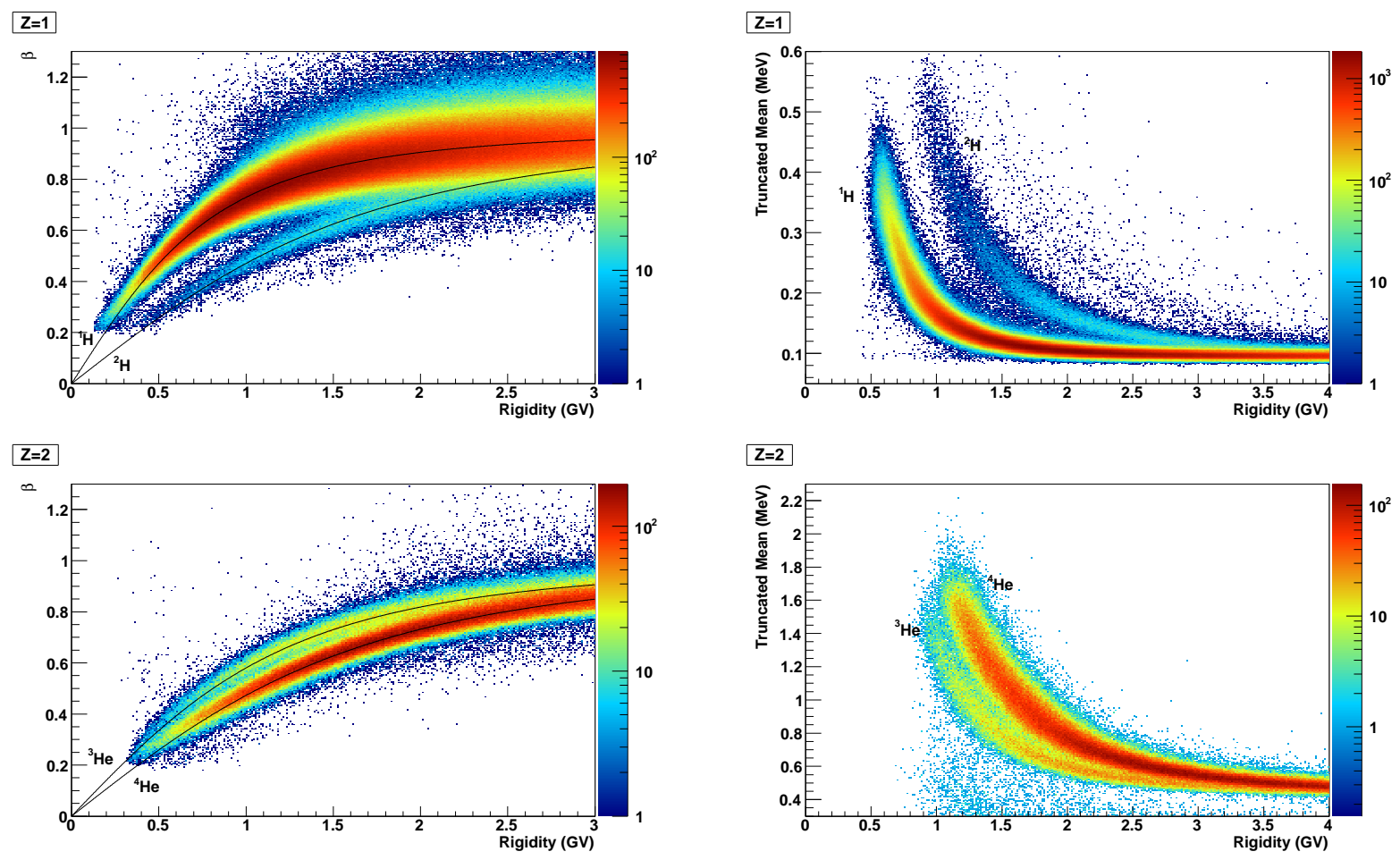

Figure 1: $\beta$ vs. rigidity for $Z=1$ (top) and $Z=2$ (bottom) particles.

Figure 2: Mass separation for $Z=1$ (top) and $Z=2$ (bottom) particles using the "truncated mean"-method.

fully penetrating the calorimeter. In the single silicon layer the energy loss distributions shows a Landau tail which degrades the resolution of the $\mathrm{dE} / \mathrm{dx}$ measurement. Using a truncation method, the $50 \%$ of samples with larger pulse amplitudes are excluded before taking the mean of the $\mathrm{dE} / \mathrm{dx}$ measurements, thus reducing the effect of the Landau tail. We put an energy dependent lower limit on the number of layers after the $50 \%$ truncation, requiring at least 5 measurements at $1 \mathrm{GV}$, going up to 10 layers at $3 \mathrm{GV}$. With this requirement the lower energy limit of our analysis is around 200 $300 \mathrm{MeV} / \mathrm{n}$ (the energy to fully penetrate the calorimeter is much higher, about $400-500 \mathrm{MeV} / \mathrm{n}$ ).

In Fig. $\square$ we show the mean $\mathrm{dE} / \mathrm{dx}$ for each event vs. the rigidity measured with the magnetic spectrometer for $Z=1$ and $Z=2$ particles. The energy loss in $\mathrm{MeV}$ was derived from the measurement in MIP using a conversion factor. In both plots the isotopic separation is clearly visible.

\subsection{Raw isotope numbers}

The isotope separation as well as the determination of isotope fluxes was performed identical to [U] in intervals of kinetic energy per nucleon. Since the magnetic spectrometer measures the rigidity of particles, this implies different rigidity intervals according to mass of the the isotope under study.

\subsubsection{Raw isotope numbers with the ToF}

The particle counts in each rigidity range were derived in a similar manner as in [U] by fitting gaussians to the $1 / \beta$ distributions as shown by the solid lines in Fig. Bl. $1 / \beta$ distributions were 

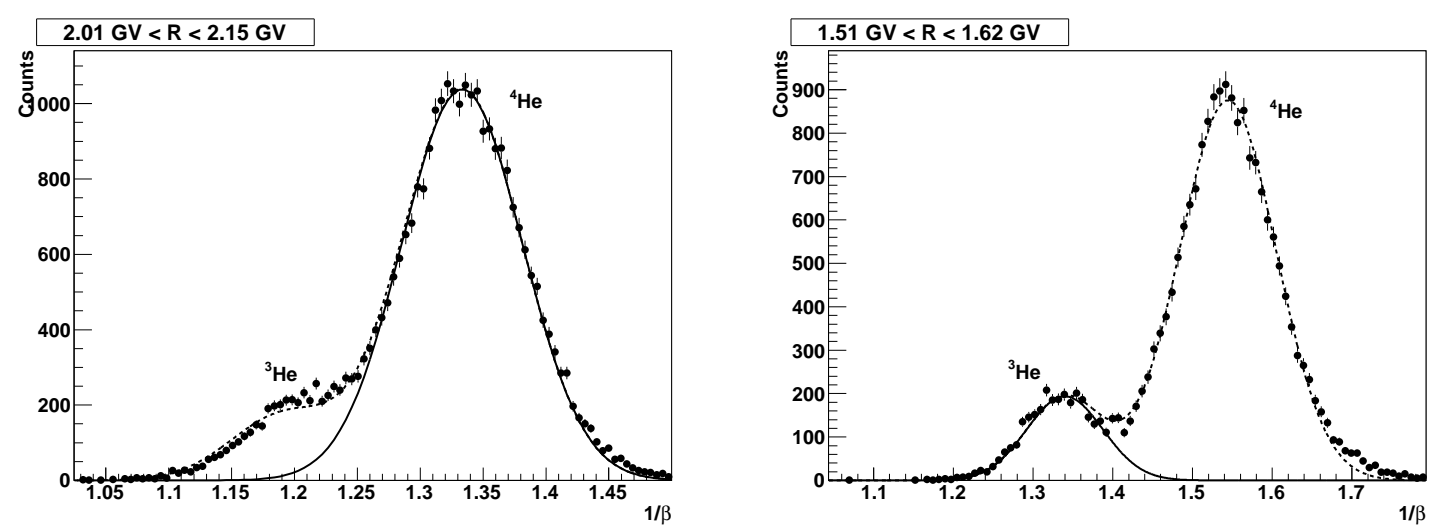

Figure 3: $1 / \beta$ distributions for helium in the $0.439-0.492 \mathrm{GeV} / \mathrm{n}$ kinetic energy range for ${ }^{4} \mathrm{He}($ left $)$ and ${ }^{3} \mathrm{He}($ right $)$. The dashed line shows the combined fit while the solid line shows the estimated ${ }^{4} \mathrm{He}$ and ${ }^{3} \mathrm{He}$ individual signals.

chosen since the shape of a $1 / \beta$ distribution is gaussian. For the fitting method the ROOT analysis package was used. When analyzing the hydrogen distributions the ${ }^{1} \mathrm{H}$ peak is well pronounced and barely affected by the shape of the neighbouring ${ }^{2} \mathrm{H}$ distribution, for that reason a single gaussian was fitted. The gaussian fit to the ${ }^{2} \mathrm{H},{ }^{3} \mathrm{He}$ and ${ }^{4} \mathrm{He}$ distributions becomes a little more complicated since the neighbouring isotopes are quite abundant and have an impact on the fitting. Consequently we applied a double gaussian fit to the histograms, see Fig. B., and the whole process was done in a more elaborated manner compared to the analysis presented in [U]. Additionally we applied a suppression procedure to the abundant neighbour, similar to the approach in [四]. To suppress the abundance of protons, we choose the energy loss measurements in the silicon layers of the tracking system and in the scintillators of the ToF versus the rigidity, both systems provide up to 12 energy loss measurements. In the $Z=2$ data the level of the ${ }^{4} \mathrm{He}$ background in the ${ }^{3} \mathrm{He}$ sample is much smaller compared to the $Z=1$ data, but similar checks like the one described above showed that also in this case a soft cut to suppress ${ }^{4} \mathrm{He}$ at higher energies based on the lowest energy release in the tracking system improved the ${ }^{3} \mathrm{He}$ selection. Note that this suppression was not used for the $Z=2$ analysis in [四]. Fig. [ 3 shows the $1 / \beta$ distributions used to select ${ }^{3} \mathrm{He}$ (right panel) and ${ }^{4} \mathrm{He}$ (left panel) in the kinetic energy interval $0.439-0.492 \mathrm{GeV} / \mathrm{n}$ corresponding to $1.51-1.62 \mathrm{GV}$ for ${ }^{3} \mathrm{He}$ and $2.01-2.15 \mathrm{GV}$ for ${ }^{4} \mathrm{He}$.

\subsubsection{Raw isotope numbers with the calorimeter}

The $d E / d x$ distributions of the calorimeter have a non-gaussian shape, therefore one has to model the expected distributions of the observable quantities and then perform likelihood fits, we used the "RooFit" toolkit for this task. First one has to create the expected $d E / d x$ distributions ("probability density function": PDF) for each isotope, this is done using the full Monte Carlo simulation of the PAMELA apparatus based on the GEANT 4 code, which has been already described in [四]. When taking the simulated energy loss in each layer as coming from GEANT 4, we noticed that the resulting PDFs showed a slight mismatch from the flight data. We found that the width of the histograms was smaller than in the real data, also there was a small offset of about $1-2 \%$. We 

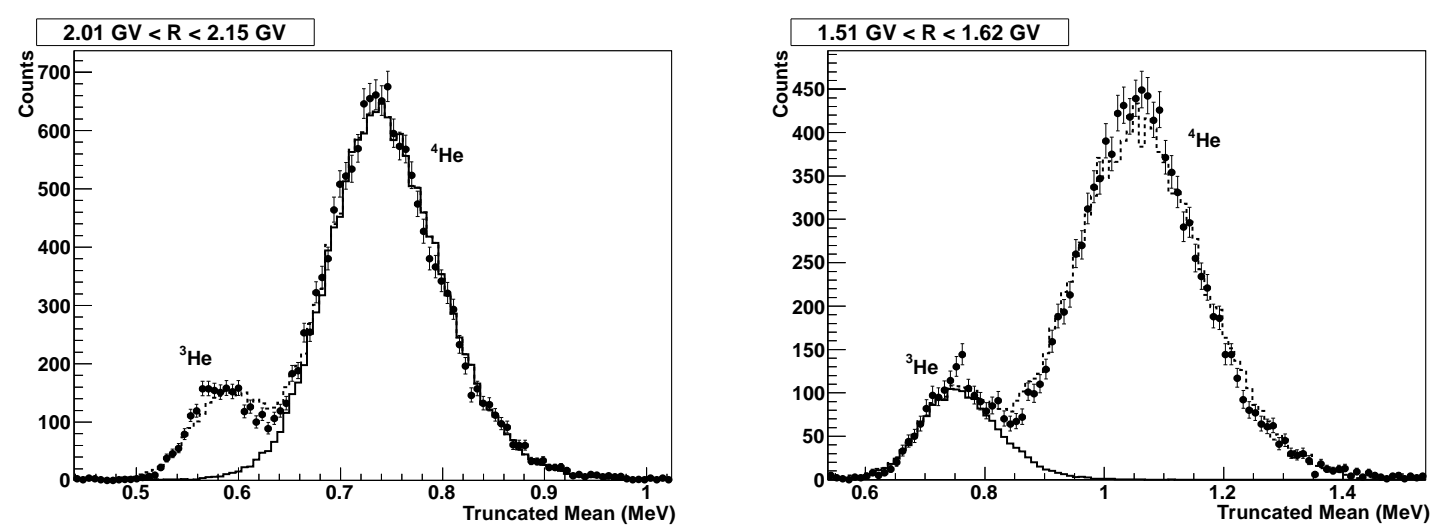

Figure 4: Example of the truncated mean distributions for helium in the $0.439-0.492 \mathrm{GeV} / \mathrm{n}$ kinetic energy range for ${ }^{4} \mathrm{He}(l e f t)$ and ${ }^{3} \mathrm{He}(r i g h t)$. The dashed line shows how the combined fit using the two PDFs derived with the modified GEANT 4 simulation matches the data points (black points), while the solid line shows the estimated ${ }^{4} \mathrm{He}$ and ${ }^{3} \mathrm{He}$ individual signals.

applied a multiplicative factor to the simulated energy loss in a layer, plus adding a gaussian spread of the signal of some percent.

As an example we show in Fig. 团 the truncated mean distributions for helium in the 0.439 - $0.492 \mathrm{GeV} / \mathrm{n}$ kinetic energy range for ${ }^{4} \mathrm{He}($ left $)$ and ${ }^{3} \mathrm{He}($ right $)$. The dashed line shows how the combined fit using the two PDFs derived with the modified GEANT 4 simulation matches the data points (black points) while the solid line shows the estimated individual ${ }^{3} \mathrm{He}$ and ${ }^{4} \mathrm{He}$ signals. The kinetic energy range is the same as shown in Fig. [l] for the ToF, the difference in the isotopic separation is clearly visible.

\subsection{Flux Determination}

To derive each isotope flux the number of selected events derived in the previous section had to be corrected for the selections efficiencies, particle losses, contamination and energy losses. Most of the corrections could be directly taken from [四], only some efficiencies in the ToF analysis were changed. A new correction is the efficiency for the calorimeter, which is is derived using simulated data for higher energies and flight data for lower energies, using the redundant detectors to select the isotopes. The selection cuts for the actual analysis were described in section 3.2.2, this approach gives an efficiency around $50-60 \%$ down to ca. $200 \mathrm{MeV} / \mathrm{n}$ where the efficiency then shows a steep decrease. As mentioned already above one can do these selections within the calorimeter analysis in various ways, but the efficiencies are very sensitive to the applied cuts. Preliminary results for a different selection cut (with an efficiency of only about $25 \%$ and a steep drop already at $300-400$ $\mathrm{MeV}$ ) have been presented in earlier publications ([ए]]).

Other corrections like the loss of hydrogen and helium nuclei due to inelastic interactions or the contribution to ${ }^{2} \mathrm{H}$ from ${ }^{4} \mathrm{He}$ inelastic scattering are taken from [四] without changes and we refer to this paper for more details. Furthermore one needs the geometrical factor and the live time of the instrument as evaluated by the trigger system, also here we refer to [四]. The finite resolution of the magnetic spectrometer and particle slowdown due to ionization energy losses results in a 
distortion of the particle spectra. A Bayesian unfolding procedure was used to derive the number of events at the top of the payload ([四], []]).

\subsection{Systematic uncertainties}

The event selection criteria described in section w. energy proton and helium fluxes [D]. In that paper the systematic errors of the selection have been studied using flight data and simulations, resulting in a quoted systematic uncertainty of ca. $4 \%$. This error is used also in this work. Other systematic uncertainties presented in [四] have been reviewed and updated to the new analysis methods when neccessary. For example, the quality of the Gaussian fit procedure in the ToF analysis was studied in detail, depending on the particle, we found a systematic uncertainty of $0.5 \%$ for low and medium energies increasing to $2 \%-5 \%$ at the highest energy bins. Other systematic uncertainties are taken from [四] without changes and we refer to this paper for more details. The total systematic uncertainties considered in this analysis are shown in Figs. 5 and 6 .

\section{Results and discussion}

In Figure $\square$ and 6 we show the hydrogen and helium isotope fluxes (top) and the ratios of the fluxes (bottom) measured with the ToF or the calorimeter, compared to other measurements [ 9 , [ ],

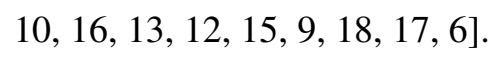

It is visible that the former results show a large spread and it is obvious that the PAMELA results are more precise in terms of statistics. In this context it is important to know that all the former measurements shown in Figures $\square$ and $\mathbf{6}$, except AMS-01, are from balloon-borne experiments and thus effected by the non-negligible background of atmospheric secondary particle production.

It is worth noting that the PAMELA results obtained via the ToF analysis and the via the multiple $d E / d x$ measurements with the calorimeter agree very well within their systematic errors. This gives confidence to the results.

The scientific interest in these isotopes of ${ }^{1} \mathrm{H},{ }^{2} \mathrm{H},{ }^{3} \mathrm{He}$ and ${ }^{4} \mathrm{He}$ are determined by the question about their origin. It is believed that the protons and the ${ }^{4} \mathrm{He}$ particles are predominantly of primary origin thus arise directly from their sources while ${ }^{2} \mathrm{H}$ and ${ }^{3} \mathrm{He}$ are of secondary origin thus are produced by interactions of these primaries with the interstellar gas. The interpretation of these results then allows to study more in detail the conditions of their propagation in the interstellar space. A comprehensive and detailed study and discussion and interpretation of our results in this context is beyond the scope of this paper, but we like to refer to a recent paper published by [0] $]$.

\section{References}

[1] O. Adriani et al. 2013 Astrophys. J. 7702

[2] O. Adriani et al. 2011, Science 33269 - Supplementary Online Material

[3] O. Adriani et al. 2009 Phys. Rev. Lett. 102051101

[4] M. Aguilar, J. Alcaraz et al. 2002 Phys. Rep. 366331

[5] M. Aguilar et al. 2011 Astrophys. J. 736105 

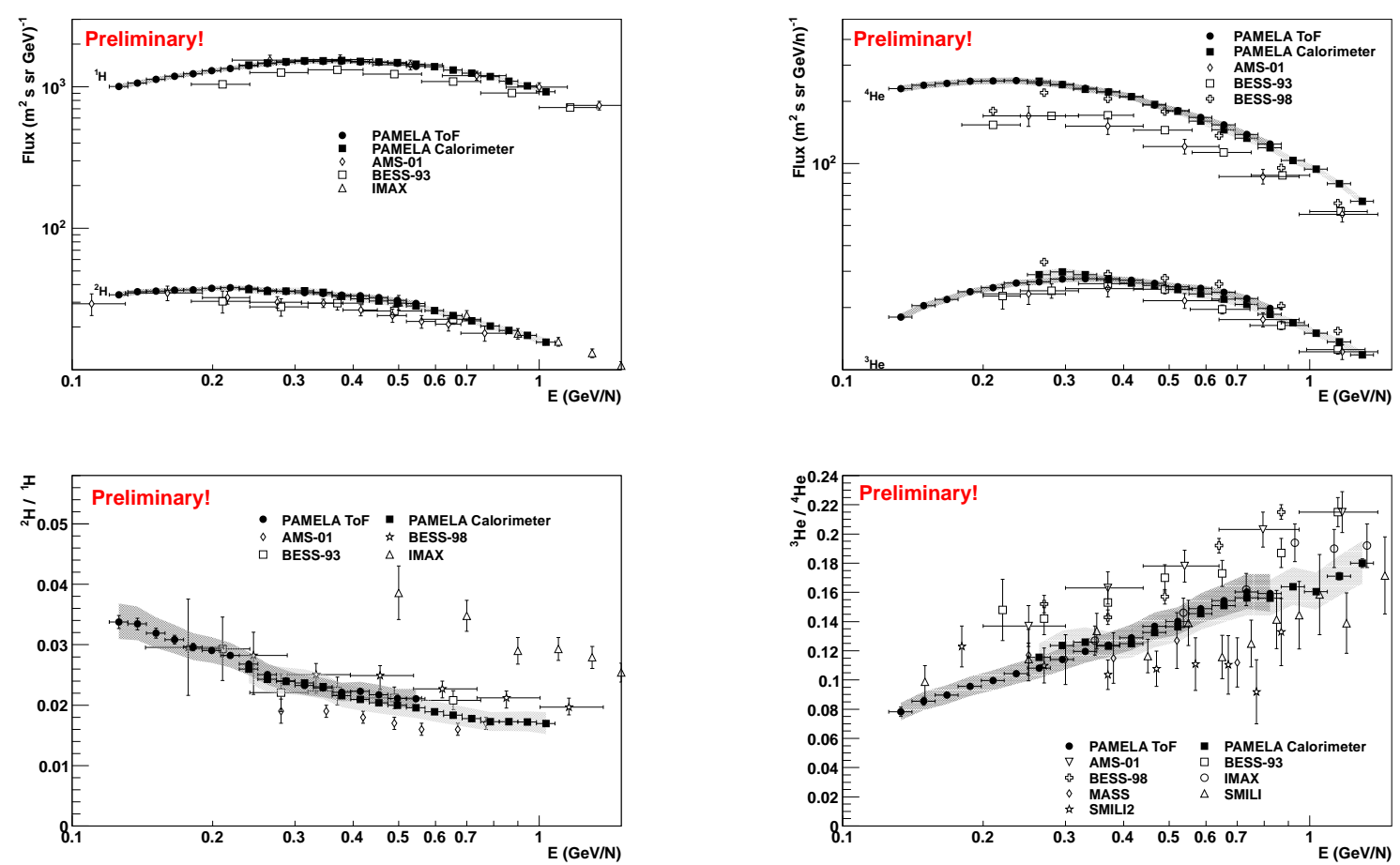

Figure 5: ${ }^{1} \mathrm{H}$ and ${ }^{2} \mathrm{H}$ absolute fluxes (a) and their ratio (b) derived with the ToF (circles) or the calorimeter (squares). Previous experiments: AMS-01 [ [ [], [1, ㅁ]], BESS-93 [ए6]], BESS-98 [ए3]], IMAX [四]. Error bars show the statistical uncertainty while shaded areas show the systematic uncertainty.

Figure 6: ${ }^{4} \mathrm{He}$ and ${ }^{3} \mathrm{He}$ absolute fluxes (a) and their ratio (b) derived with the ToF (circles) or the calorimeter (squares). Previous experiments: AMS [[]], BESS-93 [ㅁ], BESS-98 [ㅁ]], IMAX [ㄷ]], SMILI-1 [目], SMILI2[ㅇ]], MASS [ए7]. Error bars show statistical uncertainty while shaded areas show systematic uncertainty.

[6] J. J. Beatty et al. Astrophys. J. 413268

[7] M. Boezio et al. 2002 Nucl. Instr. and Meth. A 487 407-422

[8] B. Coste, L. Derome, D. Maurin \& A. Putze 2012 AAP 539 A88

[9] G. A. de Nolfo et al. 2000 AIP Conf. Proc. 528425

[10] G. Lamanna et al. 2001 Proc. 27th Int. Cosmic Ray Conf. (Hamburg) 1617

[11] W. Menn et al. 2013a J. Phys.: Conf. Ser. 409012030

[12] Z. D. Myers et al. 2001 Proc. 27th Int. Cosmic Ray Conf. (Hamburg) 1805

[13] Z. D. Myers et al. 2005 Adv. Space Res. 35 151-155

[14] P. Picozza et al. 2007 Astropart. Phys. 27296

[15] O. Reimer et al. 1998 Astrophys. J. 496490

[16] J. Z. Wang, E. S. Seo et al. 2002 Astrophys. J. 564244

[17] W. R. Webber et al. 1991 Astrophys. J. 380230

[18] J. P. Wefel et al. 1995 Proc. 24th Int. Cosmic Ray Conf. (Rome) 2630 